\title{
Macro-Kinematic Approach for Shear Behaviour of Short Coupling Beams with Conventional Reinforcement
}

\author{
Boyan I. Mihaylov ${ }^{1}$ and Renaud Franssen ${ }^{2}$ \\ ${ }^{1}$ Department of Architecture, Geology, Environment and Constructions (ArGEnCo), \\ University of Liège; Building B52, Allée de la Découverte 9, 4000 Liège, Belgium; \\ PH (32) 4-366-9497; FAX (32) 4-366-9133; email: boyan.mihaylov@ulg.ac.be \\ ${ }^{2}$ Department of Architecture, Geology, Environment and Constructions (ArGEnCo), \\ University of Liège; Building B52, Allée de la Découverte 9, 4000 Liège, Belgium; \\ PH (32) 4-366-9935; email: r.franssen@ulg.ac.be
}

\begin{abstract}
Short coupling beams in wall structures work predominantly in shear and develop complex deformation patterns. For this reason they cannot be modelled based on the classical plane-sections-remain-plane hypothesis, and are typically designed with strut-and-tie models. However, because strut-and-tie models are inherently conservative, they can result in very large amounts of shear reinforcement (stirrups), and therefore significant construction difficulties. In addition, strut-and-tie models do not provide information about the deformation capacity of coupling beams, which is important for performance-based seismic design. To address these challenges, this paper discusses a three-parameter kinematic theory (3PKT) for the shear strength and deformation patterns of short coupling beams. The 3PKT approach is situated between simple and conservative strut-and-tie models and complex non-linear finite element (FE) models. While FE models use a large number of degrees of freedom (DOFs) to describe the deformation patterns in coupling beams, the 3PKT method is based on a kinematic model with only three DOFs. The paper presents the formulation of the model and its validation with tests.
\end{abstract}

Keywords: coupling beams, shear, deformation patterns, kinematic model

\section{INTRODUCTION}

Coupling beams in shear wall structures of buildings serve to link the individual walls into a stiff lateral-load resisting system. They are located above door or window openings and feature relatively small aspect ratios ( The adjacent shear walls subject the coupling beams to double curvature bending and high shear forces. The highest shear forces typically develop in stiff short coupling beams with $a / h \leq 2.5$ that are the focus of this paper. Such members are susceptible to brittle shear failures that limit their force and deformation capacity. To prevent such failures, short coupling beams are reinforced with either conventional stirrups or diagonal reinforcement. This paper will discuss a new approach for the modelling of diagonal tension failures in short coupling beams with conventional reinforcement.

Short coupling beams do not obey the plane-sections-remain-plane hypothesis, and therefore cannot be modelled based on the classical beam theory. Instead, such 
members are typically designed with strut-and-tie models based on a clear visualisation of the flow of forces in the member (Lee et al. 2008, fib 2013). However, because strut-and-tie models are inherently conservative, they can result in very large amounts of shear reinforcement (stirrups), and therefore significant construction difficulties in the case of heavily loaded coupling beams. It is therefore the purpose of this paper to propose an alternative approach that can be used for refined calculations when necessary. Due to the similarities between short coupling beams and the inner shear spans of continuous deep beams, this approach will be derived as an extension to an earlier three-parameter kinematic theory (3PKT) for deep beams (Mihaylov et al. 2015).

\section{THREE-PARAMETER KINEMATIC THEORY FOR DEEP BEAMS}

The three-parameter kinematic theory (3PKT) was developed based on the premise that the apparently complex deformation patterns observed in the shear spans of continuous deep beams can be represented by a simple kinematic model (Mihaylov et al. 2015). For shear spans subjected to double curvature, the model uses three independent kinematic parameters (or degrees of freedom, DOFs), while for shear spans of simple beams the DOFs are reduced to only two. As coupling beams work in double curvature, they can be described similarly to the inner shear spans of continuous deep beams. The kinematic model provides conditions for compatibility of deformations, while the 3PKT also includes equilibrium equations and constitutive relationships for the mechanisms of shear resistance. The complete set of equations of the kinematic approach is summarized in Figure 1.

Figure 1a shows the kinematic model and its three degrees of freedom. In the model the shear span is divided by a critical diagonal crack that extends between the support and loading points. The regions below and above the critical crack are marked by flexure-shear cracks that outline two fans of rigid concrete struts, one centered at the loading point and the other at the support. The DOFs of the model are the average strains along the bottom and top longitudinal reinforcement $\varepsilon_{t 1, a v g}$ and $\varepsilon_{t 2, a v g}$, as well as the transverse displacement $\Delta_{c}$ in the critical loading zone of the shear span (CLZ) where the concrete crushes at failure.

As evident from the diagrams in Figure 1a, the complete deformation pattern of the shear span is represented as a superposition of two basic patterns. In the first pattern the two fans of struts open and the cracks between them widen. In the second pattern the two fans remain undeformed, while the deformations concentrate in the CLZ. As a result of the deformations in the CLZ, the critical diagonal crack undergoes additional widening as well as slip deformations. Using small-displacement kinematics, the complete displacement field of the two deformation patterns can be expressed as a function of the 3 DOFs of the kinematic model.

A key assumption of the $3 \mathrm{PKT}$ is that the shear failure is triggered by the crushing of the CLZ. This assumption has been confirmed in experimental and analytical studies. It is used to derive an expression for DOF $\Delta_{c}$ by simplifying the geometry and strain

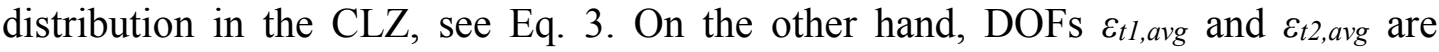
expressed with the bending moments in the end sections of the shear span (and therefore with the shear $V$ ), assuming that the longitudinal reinforcement remains 
elastic, see Eq. 1-2. To calculate these DOFs and the shear force at failure, the 3PKT requires an iterative solution procedure.

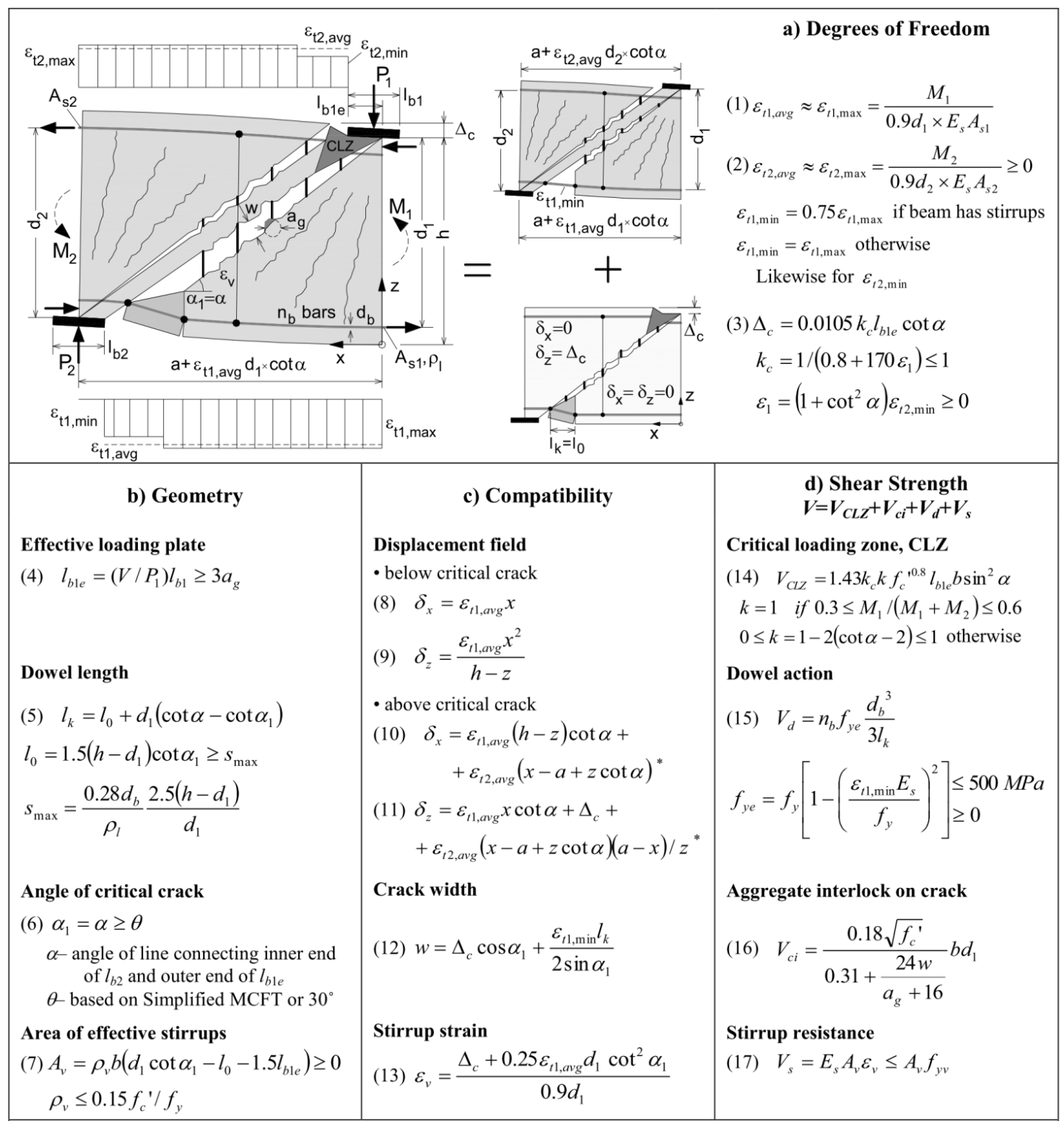

Figure 1. Three-parameter kinematic theory for deep beams (3PKT).

The solution procedure begins by calculating the geometry of the kinematic model from the equations in Figure 1b, and by assuming an initial value of the shear force $V$. With this initial value, Eq. 1-3 are used to calculate DOFs $\varepsilon_{t 1, a v g}, \varepsilon_{t 2, a v g}$, and $\Delta_{c}$. These DOFs are in turn used to calculate the width of the critical diagonal crack $w$ and the strain in the transverse reinforcement (stirrups) $\varepsilon_{v}$ from Eq. 12-13 in Figure 1c. These equations are derived directly from the kinematic model and have meaning of conditions for compatibility of deformations. In a following step, the crack width $w$ is used to estimate the shear $V_{c i}$ transferred across the critical diagonal crack by means of aggregate interlock. This calculation is based on a constitutive relationship proposed by Vecchio and Collins (1986), see Eq. 16 in Figure 1d. Similarly, strain $\varepsilon_{v}$ 
is used to evaluate the stress in the stirrups, and therefore the shear resistance $V_{s}$ provided by the stirrups, see Eq. 17. For simplicity, it is assumed that the stress-strain relationship of the stirrups is elastic-perfectly plastic. In addition to the shear components $V_{c i}$ and $V_{s}$, a significant portion of the shear in deep beams is carried by the critical loading zone. Similarly to DOF $\Delta_{c}$, shear component $V_{C L Z}$ is derived by assuming that the concrete in the CLZ is at crushing when the beam fails, see Eq. 14. Finally, the last component of shear resistance is attributed to the dowel action of the longitudinal reinforcement $V_{d}$ which is typically relatively small (Eq. 15). Having calculated the four components of shear resistance, a new value of the shear force $V$ is obtained by adding up these components. With the updated shear force, the calculations return to Eq. 1-3 for the DOFs of the kinematic model, and the procedure is repeated until $V$ converges to a constant value. This value represents the shear strength prediction of the 3PKT. The predicted DOFs can then be used to calculate the complete displacement field of the shear span at failure by using Eq. 8-11.

\section{EXTENDED KINEMATIC APPROACH FOR SHORT COUPLING BEAMS}

As mentioned earlier, the 3PKT can be applied to short coupling beams because they work in double curvature similarly to the inner shear spans of continuous deep beams. The main difference between coupling beams and deep beams is the manner in which the forces are introduced in the shear span. While deep beams are typically loaded by columns, coupling beams are loaded by the adjacent shear walls, see Figure 2. This requires certain modifications of the original approach mainly related to the critical loading zone. It can be shown that in deep beams the size of the CLZ, and therefore its strength $V_{C L Z}$ and displacement capacity $\Delta_{c}$, are proportional to the size of the loading column $l_{b l}$, see Figure 1. More specifically, $V_{C L Z}$ is proportional to the effective size of the loading element $l_{b l e}$ expressed by Eq. 4. However, because the CLZ of coupling beams does not merge into a column with a fixed width but into a large shear wall, the effective size $l_{b l e}$ is not clearly defined. A similar situation arises in the base of short shear walls where the CLZ of the wall merges into a stiff foundation. In the kinematic modelling of short shear walls Mihaylov et al. (2016) have proposed to estimate $l_{b l e}$ as a percentage of the length of the wall diagonal. The same approach is adopted here by using the length of the diagonal of the coupling beam:

$$
l_{b 1 e}=0.11 \sqrt{a^{2}+h^{2}}
$$

where the coefficient 0.11 is the same as that proposed for the modelling of walls.

In addition to this modification, it should also be taken into account that coupling beams are typically subjected to symmetrical double curvature bending and feature symmetrical top and bottom longitudinal reinforcement. As a consequence, DOFs $\varepsilon_{t l, a v g}$ and $\varepsilon_{t 2, a v g}$ are equal and the $3 \mathrm{PKT}$ can be viewed as a $2 \mathrm{DOF}$ method. Equations 1-2 of the original 3PKT can therefore be replaced by a single equation:

$$
\varepsilon_{t, \text { avg }} \approx \varepsilon_{t, \text { max }} \approx \varepsilon_{t, \text { min }}=\frac{V(a / 2)}{0.9 d \times E_{s} A_{s}}
$$

Finally, the last modification of the original 3PKT method concerns the shear carried by the transverse reinforcement $V_{s}$, and more specifically the area of the stirrups $A_{v}$ 
that contribute to $V_{s}$. Equation 7 for $A_{v}$ accounts for the fact that, due to the relatively small stirrup strains near the ends of the shear span of deep beams, not all stirrups crossing the critical diagonal crack are effective in resisting the shear force. However, experimental studies of coupling beams have shown that the stirrups are almost fully activated (Paulay $(1969,71$, ) and therefore Eq. 7 is replaced by:

$$
A_{v}=\rho_{v} b \times 0.9 d \cot \alpha_{1}
$$

where the angle of the critical crack is estimated as

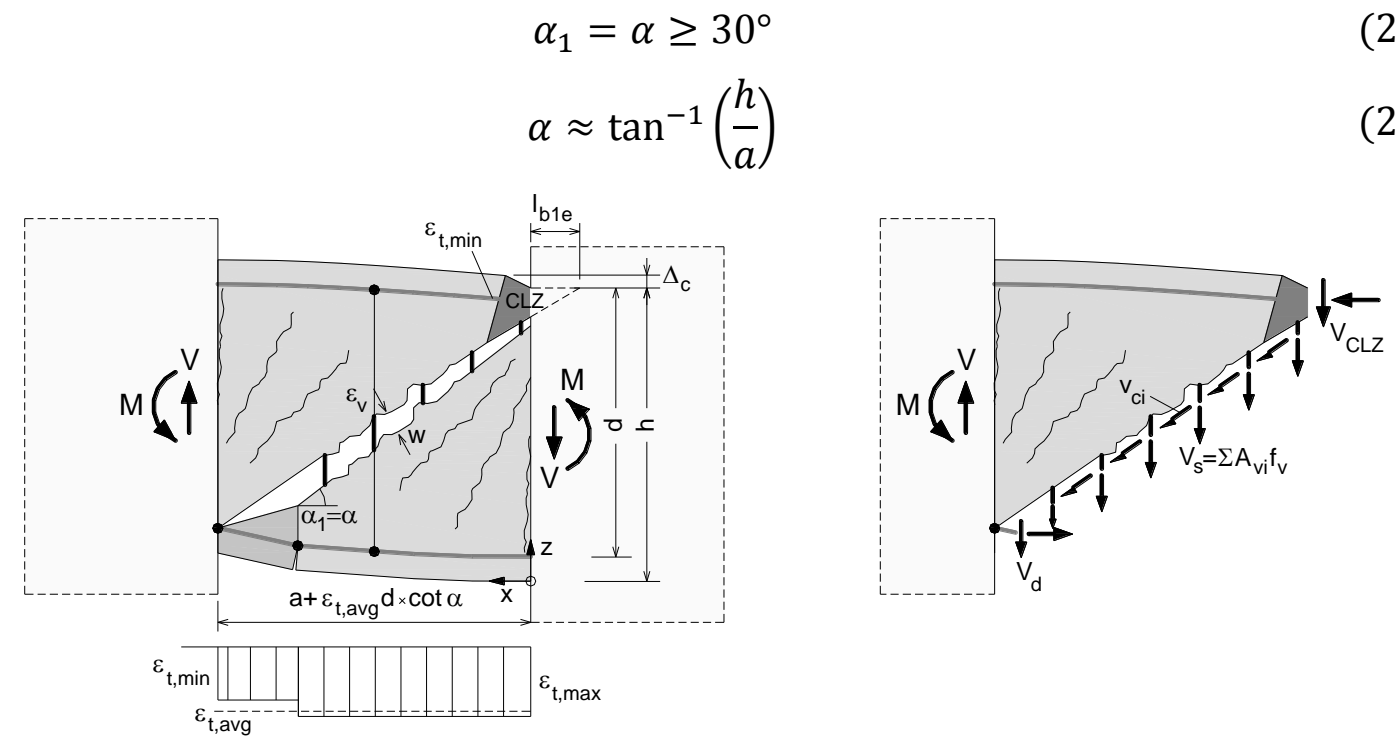

Figure 2. 3PKT for short coupling beams.

\section{COMPARISONS WITH TESTS}

The proposed extended 3PKT method is applied to a short coupling beam tested by Paulay $(1969,71)$. The test specimen, named beam 391, had a $152 \mathrm{~mm}$ by $991 \mathrm{~mm}$ rectangular section and a shear-span-to-effective-depth ratio $a / d=1016 / 991=1.11$. The longitudinal reinforcement of the beam consisted of symmetrical top and bottom bars with a ratio $\rho=1.06 \%$, while the transverse reinforcement consisted of stirrups with a ratio $\rho_{v}=0.88 \%$. The complete set of properties of the specimen is provided in Table 1 . Beam 391 was subjected to monotonic loading until it failed at a shear force of 777 $\mathrm{kN}$ along a critical diagonal crack, see Figure 3 . The shear strength of the beam can be predicted based on the 3PKT method for coupling beams by using the iterative solution procedure described earlier. However, to demonstrate the method more clearly, the solution of the 3PKT equations is presented in a graphical form in Figure 3. On the horizontal axis of the plot is the unknown DOF $\varepsilon_{t, \text { avg }}=\varepsilon_{t 1, \text { avg }}=\varepsilon_{t 2 \text {,avg }}$ while on the vertical axis are the shear forces. For each value of $\varepsilon_{t, a v g}$, the shear on the beam can be calculated from Eq. 19 based on the moment equilibrium of the shear span. This shear force can be seen as a demand on the shear span which at failure must equal the shear resistance along the critical diagonal crack. The shear resistance is obtained as the sum of the four shear components expressed from Eq. 14-17 and plotted in Figure 3 (curves $V_{C L Z}, V_{c i}, V_{s}, V_{d}$ ). As can be seen from the plot, the shear resistance $\Sigma V_{i}$ decreases gradually with increasing strain $\varepsilon_{t, a v g}$, while the shear 
demand increases linearly due to the linear behaviour of the longitudinal reinforcement. The solution of the 3PKT equations lies at the intersection of the two curves where the shear forces are in equilibrium. Based on this approach, the predicted value of shear resistance is $776 \mathrm{kN}$, almost exactly equal to the experimental value in this case.

Table 1. Tests of short coupling beams.

\begin{tabular}{ccccccccccccc}
\hline $\begin{array}{c}\text { beam } \\
\#\end{array}$ & $a / d$ & $b$ & $d$ & $h$ & $\rho_{l}$ & $f_{y}$ & $f_{c}^{\prime}$ & $\rho_{v}$ & $f_{y v}$ & $\begin{array}{c}\text { Load } \\
\text { type }\end{array}$ & $\begin{array}{c}V_{\text {exp }} \\
(\mathrm{kN})\end{array}$ & $\begin{array}{c}V_{\text {exp }} \\
V_{\text {pred }}\end{array}$ \\
\hline 391 & 1.11 & 152 & 917 & 991 & 1.06 & 316 & 31.5 & 0.88 & 407 & mono & 777 & 1.00 \\
392 & 1.11 & 152 & 917 & 991 & 1.06 & 316 & 37.6 & 0.88 & 407 & cyclic & 745 & 0.92 \\
311 & 1.42 & 152 & 714 & 787 & 1.58 & 313 & 36.7 & 0.88 & 386 & mono & 651 & 0.97 \\
241 & 2.27 & 152 & 536 & 610 & 1.82 & 321 & 24.2 & 0.41 & 265 & mono & 283 & 1.18 \\
\hline
\end{tabular}
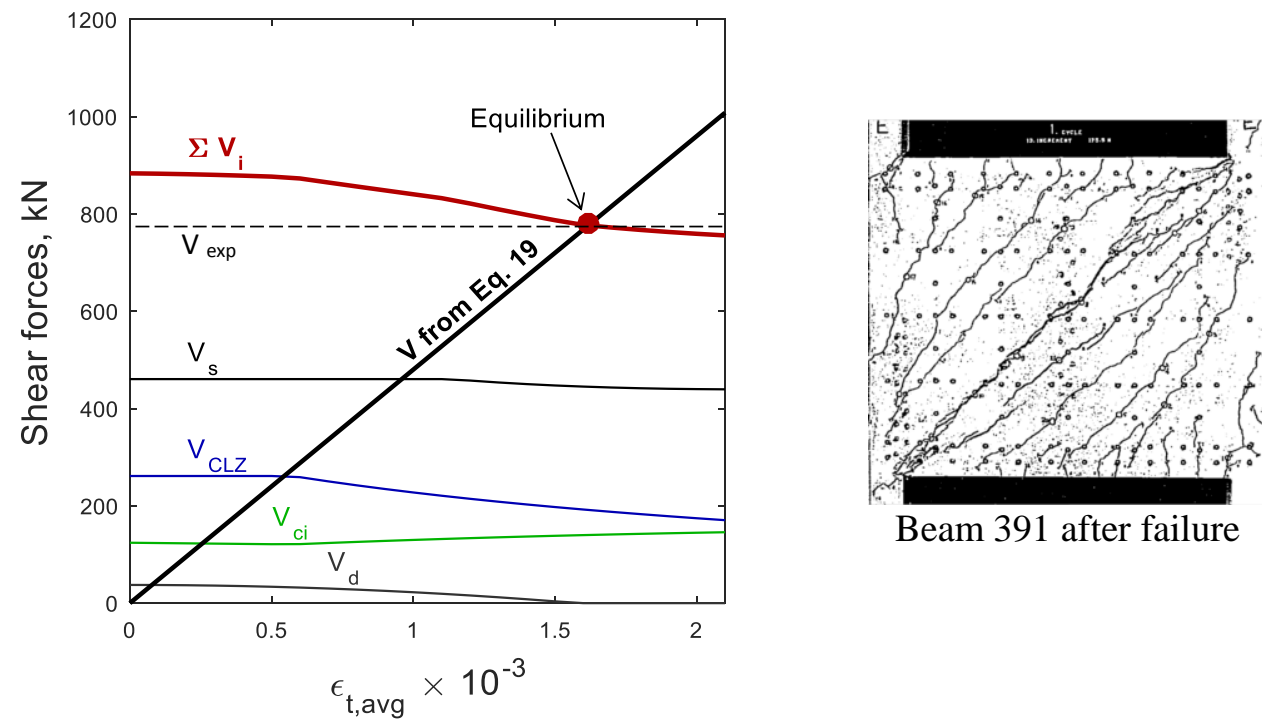

Figure 3. 3PKT applied to coupling beam 391 (Paulay 1969, 71).

Similar calculations were performed for three other coupling beams from the same experimental program that failed in shear without (or with limited) yielding of the flexural reinforcement. The results from these comparisons are summarized in Figure 4 and Table 1 . The main difference between the beams was the $a / d$ ratio which varied from 1.11 to 2.27 . Figure 4 shows the complete envelopes of the shear force versus chord rotation responses of the tests specimens. As it can be expected, the shortest coupling beam was significantly stronger than the longest one. Beams 391 and 392 were nominally identical, but the latter beam was subjected to reversed cyclic loading. Based on this comparison, the load reversals resulted in a shear strength degradation of only $4 \%$. It should be noted however that the cyclically-loaded specimen had higher concrete strength than its monotonic companion beam (37.6 vs. 31.5 MPa). As evident from the triangular markers in Figure 4, the 3PKT predicted almost exactly the strength of the monotonic test and slightly underestimated that of the cyclic test. Similarly adequate predictions were also obtained for the two longer tests specimens which were tested under monotonic load. While these results are 
promising, further tests featuring diagonal tension failures prior to flexural yielding are needed to extend the validation of the proposed approach.
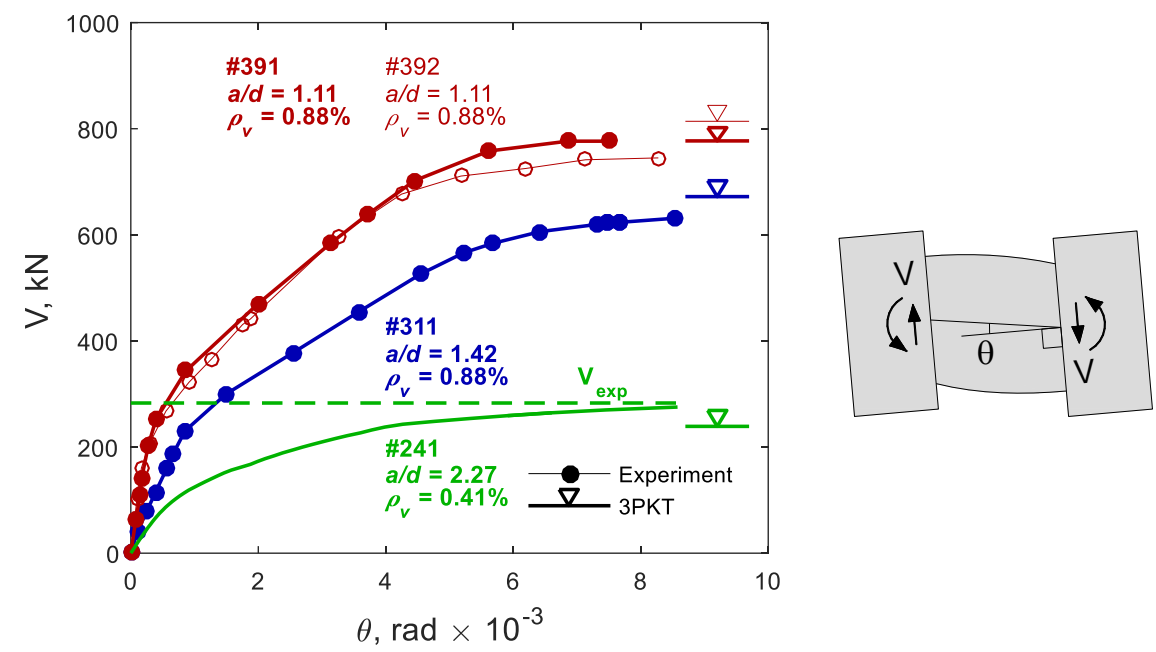

Figure 4. 3PKT comparisons with tests by Paulay $(1969,71)$.

\section{PARAMETRIC STUDY}

Finally, it is of interest to use the proposed method to study the effect of different tests variables on the shear resistance of coupling beams. In particular, it is of interest to study the effect of transverse reinforcement ratio and concrete strength, see Figure 5. Considering that the dimensions of coupling beams in high-rise buildings are typically limited due to architectural requirements, these two parameters are the main options available to engineers to increase the shear resistance of the beams.
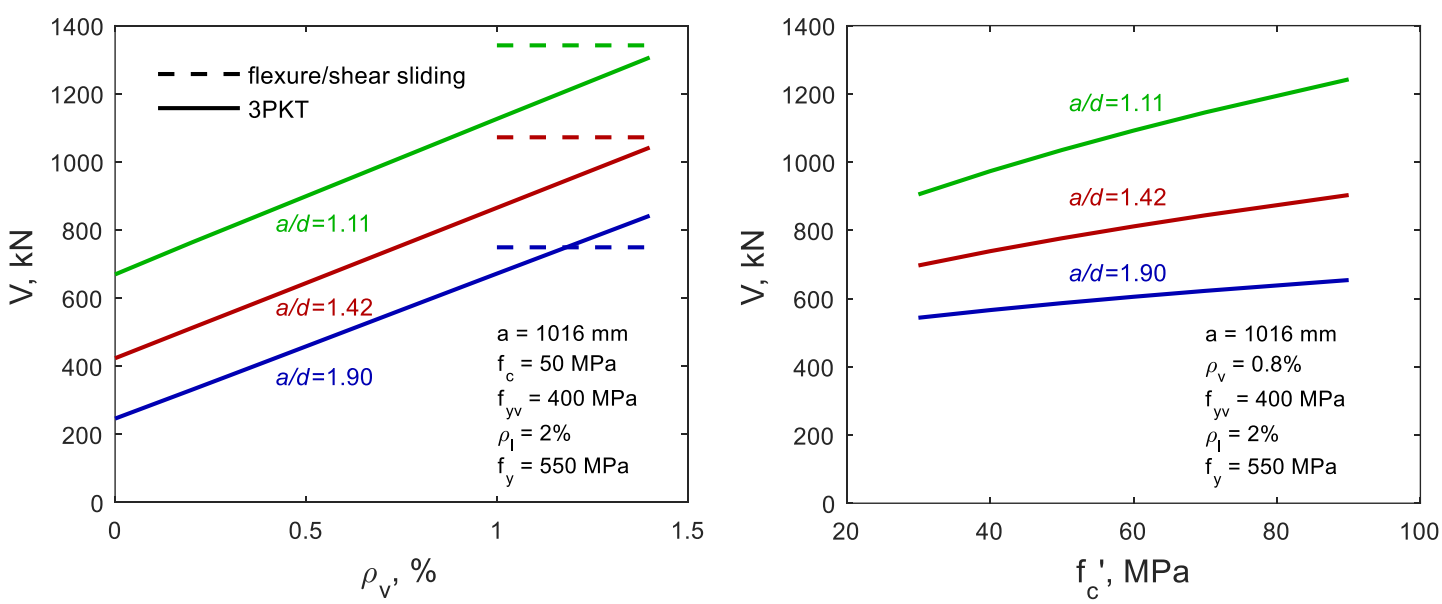

Figure 5. Effect of stirrup ratio and concrete strength on shear strength

As evident from Figure 5, the 3PKT method predicts an almost linear increase of shear strength with increasing $\rho_{v}$. For members with $a / d$ of 1.11 similar to test specimen 391 , the increase of $\rho_{v}$ from the minimum value of $0.2 \%$ to $1 \%$ results in a $48 \%$ increase of shear resistance. This trend however cannot be extended, because the capacity of the beam will eventually be limited by either flexural or sliding shear 
failure in the end sections of the beam. It can also be seen from Figure 5 that the increase of shear strength with increasing concrete compressive strength is slightly non-linear. According to the 3PKT predictions, increasing $f_{c}$ is a more effective measure in shorter beams than in longer members. This is consistent with the fact that shorter beams carry more shear via direct diagonal compression (or strut action).

\section{CONCLUSIONS}

The approach presented in this paper for calculating the shear strength of short coupling beams is an extension of a three-parameter kinematic theory for continuous deep beams. The method is aimed at predicting brittle shear failures occurring along critical diagonal cracks based on a 3DOF kinemaic description of the deformation patterns of coupling beams. Only shear failures occurring prior to yielding of the flexural reinforcement are captured by the method. The following conclusions can be drawn based on the derivations and comparisons performed in the paper:

1) The apparently complex ultimate shear behaviour of coupling beams can be modelled by using a kinematic model with only 3 DOFs;

2) The main modification required as compared to deep beams concerns the size of the critical loading zones. While in deep beams this size is proportional to the size of the loading columns, in coupling beams it can be related to the length of the diagonal of the member.

3) The proposed method predicted adequately the shear strength of four test specimens tested under monotonic and cyclic load.

4) Further comparisons with tests and numerical simulations are needed to confirm the effectiveness of the method.

\section{REFERENCES}

Fib International Federation for Structural Concrete (2013). "fib Model Code for Concrete Structures 2010.” Ernst \& Sohn, Berlin, Germany.

Lee O.J., Kuchma D.A., Baker W., Novak L.C. (2008). "Design and Analysis of Heavily Loaded Reinforced Concrete Link Beams for Burj Dubai." ACI Struct. J., 105(4), 451-459.

Mihaylov B., Hannewald P., and Beyer K. (2016). "Three-Parameter Kinematic Theory for Shear-Dominated Reinforced Concrete Walls." J. Struct. Eng., 142(7), 10.1061/(ASCE)ST.1943-541X.0001489.

Mihaylov B.I., Hunt B., Bentz E.C., Collins M.P. (2015). "Three-Parameter Kinematic Theory for Shear Behavior of Continuous Deep Beams." ACI Struct. J., 112(1), 47-57.

Paulay T. (1969). "The Coupling of Shear Walls." PhD dissertation, University of Canterbury.

Paulay T. (1971). "Coupling Beams of Reinforced Concrete Shear Walls.” ASCE J. of the Struct. Div., 97(3), 843-862.

Vecchio F.J., Collins M.P. (1986) "The Modified Compression Field Theory for Reinforced Concrete Elements Subjected to Shear," ACI Struct. J., Proceedings, 83(2), 219-231. 